\title{
并联踝康复机器人的设计与运动性能评价*
}

\author{
李剑锋 张 凯 张雷雨 张子康 左世平 \\ (北京工业大学机械工程与应用电子技术学院 北京 100124)
}

\begin{abstract}
摘要: 对于足下垂和马蹄足内/外翻患者, 均需对踝关节进行大量、重复性的康复训练, 踝康复机器人是弥补人工康复训练不 足、降低医师工作强度的关键设备。将踝关节的解剖结构及运动特性考虑在内, 提出并研制 2-UPSS/RRR 型并联踝康复机器 人样机, 可保证人-机转动中心近似重合。同时, 搭建了作用力/矩信息采集平台, 实现踝关节的被动、主动及人机交互训练, 提高了机器人、患者及医师之间的交互能力。建立了踝康复机器人的运动学模型, 通过求解其位置逆解, 得到了机器人的理 论工作空间。借助机器人样机的 3 转动自由度, 精确地测量得到踝关节全域范围内的生理运动范围。通过机器人样机的全范 围自主运动, 记录并获得装置的有效工作空间, 机器人有效工作空间小于理论工作空间, 有效工作空间的测量具有较大的实 际意义。进一步, 对比分析机器人样机的有效工作空间和踝关节生理运动范围可知, 两者在踝全域运动范围内的吻合度可达 95\%, 较好地保障了踝关节康复所需要的运动空间。基于并联踝康复机器人的量纲一速度雅可比矩阵, 对机器人的可操作度、 灵巧性等运动学性能进行了分析, 结果表明踝康复机器人在工作空间内不存在奇异位形且具有良好的运动学性能。
\end{abstract}

关键词: 并联机构; 踝康复机器人; 工作空间; 运动学性能; 灵活性

中图分类号: TP24

\section{Design and Kinematic Performance Evaluation of Parallel Ankle Rehabilitation Robot}

\section{Jianfeng ZHANG Kai ZHANG Leiyu ZHANG Zikang ZUO Shiping}

(College of Mechanical and Electrical Engineering, Beijing University of Technology, Beijing 100124)

\begin{abstract}
The ankle rehabilitation exoskeleton is a critical equipment for foot drop and talipes valgus patients requiring extensive and repetitive rehabilitation training to make up deficiencies of the manual rehabilitation training and reduce the workload of rehabilitation physicians. According to the anatomical structure and motion characteristics of the ankle, an ankle rehabilitation robot with 2-UPSS $\underline{R} R R$ configuration has been proposed and developed which can realize the approximate coincidence between the centers of the ankle and the rehabilitation robot. Meanwhile, a force/torque information collection platform is constructed to improve the interactivity among the robot, patients and physicians. The kinematic model of this rehabilitation robot is established. The corresponding theoretical workspace is obtained through solving inverse kinematics. The physiological range of activity of the ankle is accurately measured in the whole movement area with the help of the 3-degree-of-freedom of the prototype. According to the autonomous motion of the prototype, the effective workspace is recorded and obtained. The effective workspace of the robot is less than the theoretical workspace. Furthermore, the results show that the percent of contact area in the effective workspace and the physiological range can reach $95 \%$ and the rehabilitation robot can provide enough space for the injured ankle. Finally, based on the velocity Jacobian matrix of the robot, the kinematic performance such as the maneuverability and dexterity is acquired. The results show that this rehabilitation robot has no singular position and processes good kinematic performance in the effective workspace.
\end{abstract}

Key words: parallel mechanism; ankle rehabilitation robot; workspace; kinematic performance; dexterity

\section{0 前言}

随着我国老龄化加剧和交通事故频发, 脑卒中

* 国家自然科学基金(51675008,51705007)、北京市自然科学基金(3171001, 17L20019)、北京市教委科技计划(KM201810005015)、中国博士后基金 特别资助(2018T110017)和北京市博士后基金(2017-ZZ-038)资助项目。 20180710 收到初稿, 20181207 收到修改稿
发病率逐步升高，高比例患者存在因足下垂导致下 肢运动功能障碍 ${ }^{[1-3]}$ 。另外, 马蹄足内/外翻亦属于 我国少年、儿童的骨科多发病, 需进行踝关节松解、 矫形手术治疗。为改善下肢运动功能和步态重建, 上述患者均需对踝关节进行大量、重复性的康复训 练 ${ }^{[4]}$ 。传统的康复训练疗法是康复治疗师对患者进 行一对一徒手康复治疗，不足之处是效率低下、劳 动强度大、缺乏科学有效的数据监测与反馈, 难以 
对患者的康复状况进行数值化评价。

为弥补上述不足, 国内外院校相继开展踝康复 机器人的相关研究工作。早期的踝康复机构 ${ }^{[5-6]}$ 具有 1 2 个自由度、结构简单, 存在自由度不足导致踝 关节难以全域内运动。基于 Stewart 平台的 6 自由度 Rutgers Angle $^{[7]}$, 采用虚拟现实、力反馈等技术, 使 康复训练充满浸入感, 且可远程控制、评估、指导 患者的日常训练, 训练过程伴随小腿的移动, 若小 腿随动不及时, 易造成二次损伤, 且该系统结构复 杂, 成本较高。为了简化踝康复装置的结构和体积, SAGLIA 等 ${ }^{[9]}$ 提出一种 2 自由度踝康复机构, 由三 条支链、一条中心支撑杆和动、定平台组成, 利用 穴余驱动消除机构奇异性, 提高了工作空间和运动 性能, 但仅能够完成踝关节趾屈/背伸、内翻/外翻动 作的康复训练。戴建生等 ${ }^{[10]}$ 分别研制了 $3-\mathrm{SPS} / \mathrm{S}$ 和 3-SPS/SP 构型的康复机构, 两机构均含有一条中心 约束支链, 限制其移动自由度, 能够满足踝康复训 练的需求, 对机构分别进行了运动学和刚度分析。 刘更谦等 ${ }^{[11]}$ 、赵铁石等 ${ }^{[12]}$ 先后提出一种 3 自由度的 3-RSS/S 康复机构, 刘更谦等求解了机构的位置逆 解和雅可比矩阵, 赵铁石等用边界搜索法对机构的 理论工作空间进行了分析, 并给出了驱动电动机的 速度曲线。边辉等 ${ }^{[13]}$ 提出了 $4-\mathrm{UP}(\mathrm{Pe}) \mathrm{S} / \mathrm{S}$ 生物融合 式踝康复机器人, 由简化为球副的踝关节和机械本 体共同构成, 能够实现以踝关节为转动中心的康复 训练, 利用踝关节限制其移动自由度。汪从哲等 ${ }^{[14]}$ 提出一种 3-RUS/RRR 踝康复机构, 分析了其工作空 间、奇异性等运动学性能, 通过引入穴余驱动, 有 效地避免机构奇异性和提高其运动性能。

基于上述研究和分析, 将踝关节运动属性考虑 在内, 提出并研制一种 3 转动自由度的 2-UPS/ RRR 踝康复机器人, 具有人-机转动中心近似重合、结构 简单的优点。通过求解其机构的位置逆解和建立相 应的雅可比矩阵, 得到其理论工作空间; 将其 UPS 支链拆开, 借助康复机器人的 3 转动自由度, 检测 踝关节的生理运动范围, 同时, 结合康复机器人样 机的全范围自主运动, 测量其有效工作空间, 通过 对比三者的工作空间范围, 分析人-机运动空间的吻 合度。在此基础上, 对机器人在有效工作空间内的 可操作度、灵巧性等运动学性能进行评价。

\section{1 踝康复机器人的设计}

踝关节由胫、腓骨下端和距骨滑车构成, 踝关 节是屈戍关节, 主要功能为负重, 运动形式有背伸/ 趾屈、内收/外展、内翻/外翻等 ${ }^{[15-16]}$ 。根据踝关节运
动形式及特点, 可将踝关节的运动描述为绕 $x_{\mathrm{an}} 、 y_{\mathrm{an}}$ 和 $z_{\mathrm{an}}$ 轴方向转动, 如图 1 所示。

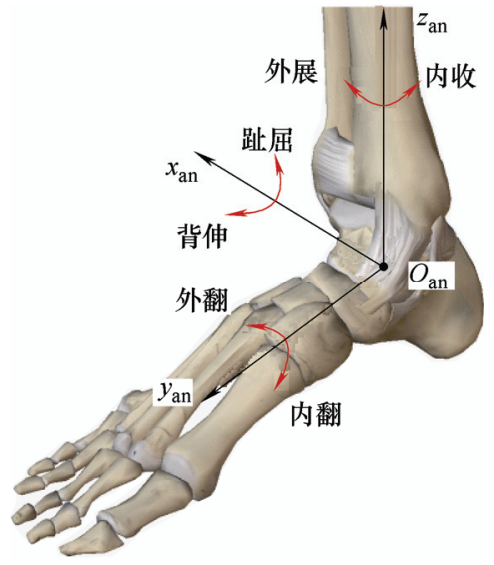

图 1 踝关节的解剖结构

为避免几余支链, 踝康复机器人的机构构型包 含 2 条驱动支链和 1 条约束支链, 约束支链由 3 个 正交的转动副 $\mathrm{R}_{1} 、 \mathrm{R}_{2} 、 \mathrm{R}_{3}$ 串联(等效球副)而成, 其 中 $\underline{R}_{1}$ 为主动运动副, 其余两条支链构型有多种选 择 ${ }^{[17]}$ 。若支链具有 6 个或以上自由度, 对动平台不 提供约束 ${ }^{[18]}$, 为保证支链结构紧凑, 支链包含 3 个 运动副, 共 6 个自由度, 基于 Kutzbach-Grübler 计 算公式, 机构的自由度数

$$
F=6(n-g-1)+\sum_{i=1}^{g} f_{i}
$$

式中, $n$ 为机构杆件数(包含基座和动平台), $g$ 为机 构运动副数目, $f_{i}$ 为第 $i$ 个运动副的自由度数。根据 机构构型, 杆件数 $n=8$, 各支链含 3 个运动副, $g=9$, 2 条驱动支链各含 6 个自由度, 约束支链含 3 个自 由度, $\sum_{i=1}^{9} f_{i}=15$, 因此, 踝康复机构自由度为

$$
F=6 \times(8-9-1)+15=3
$$

自由度数与踝关节运动学模型相符。各驱动支链包 含 1 个主动副和 2 个被动副, 综合支链构型如表 1 所示。

表 1 驱动支链构型综合

\begin{tabular}{ccc}
\hline 移动副数量 & 支链运动副组成 & 组合方式类别 \\
\hline 0 & URS、UUU & 12 \\
1 & UPS、CRS、UCU & 18 \\
2 & CPS & 6 \\
\hline
\end{tabular}

由于 UPS 支链结构简单、质量较轻、驱动方式 灵活, 采用 UPS 支链作为驱动支链, 提出一种 2-UPS/RRR 踝康复机构, 含 3 个转动自由度, 转动 副轴线正交于点 $o_{0}$, 即为其转动中心, 其机构简图 如图 2 所示。踝康复机构不含午余驱动支链, 避免 
了支链间的干涉, 结构简单, 降低了制造成本。

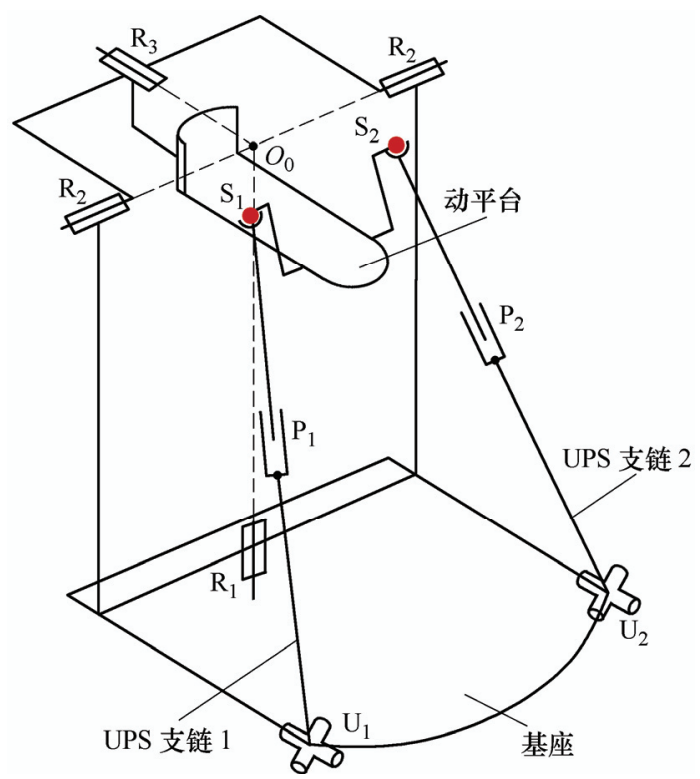

图 2 踝康复机构简图

踝康复机器人结构如图 3 所示, 采用电动推杆 驱动两个移动副 $\underline{\mathrm{P}_{i}}$, 步进电动机驱动转动副 $\underline{\mathrm{R}}_{1}$, 为 了实时监测其 3 转动自由度的角度、角速度等, 在 各转动副处安装了角度编码器, 编码器 1 \#采用齿轮 啮合的方式测量转动副 $\mathrm{R}_{1}$ 的运动学信息, 编码器 2\#、3\#直接与转动副 $R_{2} 、 R_{3}$ 的转轴串联, 通过采集 各编码器的角度, 实时得到动平台姿态角参数。控 制系统基于采集到的角度信息, 控制电动推杆和步 进电动机运动速度, 进而实现对机器人的闭环运动 控制。动平台由上、下平台组成, 如图 4a 所示, 患 者将待康复的脚掌放于上平台, 脚后跟抵靠挡板, 根据脚掌尺寸调整挡板的前后位置, 同时, 通过增 减上平台上的定制鞋垫数量调节脚掌在高度方向的 位置, 以此确保人-机转动中心近似重合。利用弹性 绑带将脚掌绑缚于上平台, 避免人-机之间出现较大

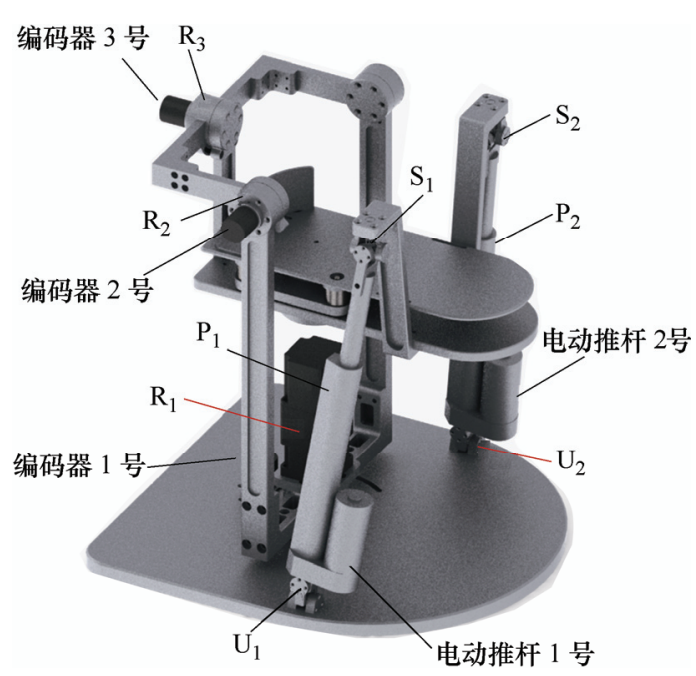

图 3 踝康复机器人的组成
的滑动、偏移等情况, 保持人-机之间具有较好的连 接强度和作用力传递性能。

为了采集人-机之间的作用力/矩, 在动平台上 增加了 4 个拉压力传感器和 1 个扭矩传感器, 如图 $4 \mathrm{~b}$ 所示, 拉压力传感器用于采集脚掌对上平台的 拉/压力, 判断踝关节背伸/趾屈、内翻/外翻所产生 的作用力/距。同时, 为了克服拉压力传感器无法测 量踝内收/外展扭矩的问题, 增加了扭矩传感器, 与 上平台通过螺纹连接的方式固连, 实现检测踝内收/ 外展的扭矩, 研制的机器人样机如图 5 所示。

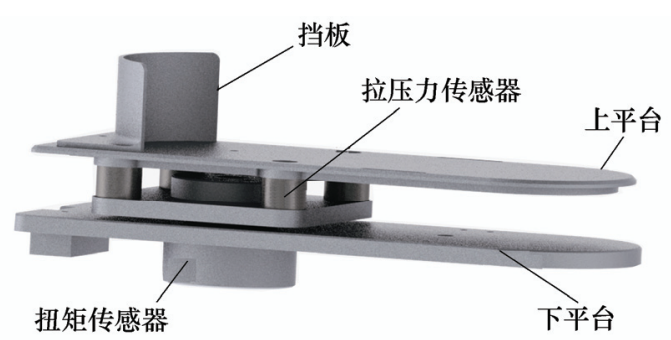

(a) 动平台的整体结构

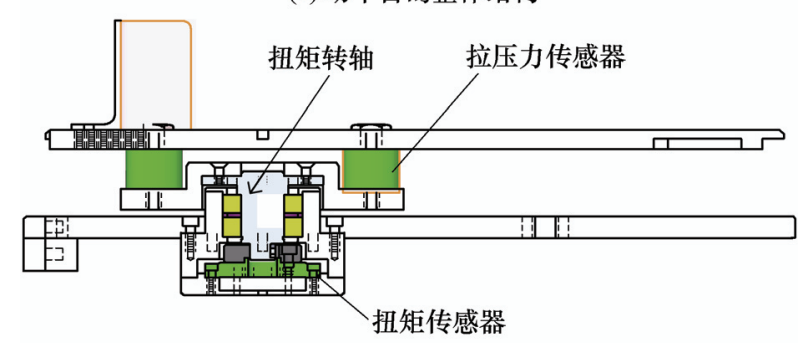

(b) 力 / 矩信息采集系统

图 4 动平台的组成

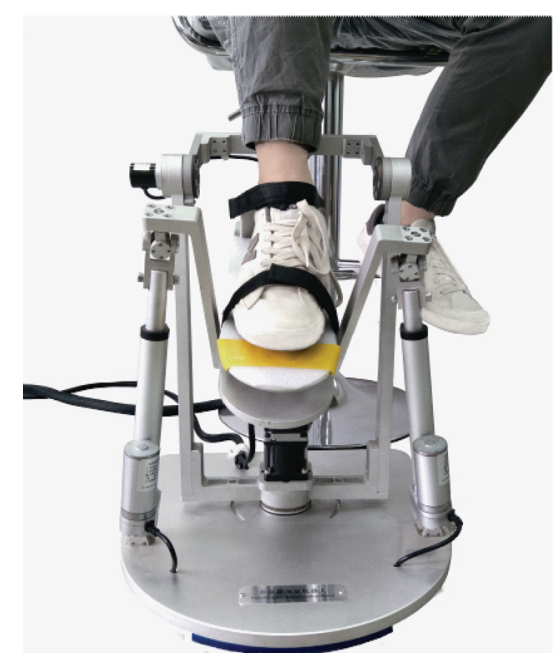

图 5 踝康复机器人样机

踝康复机器人的训练模式可分为被动训练、主 动训练及人机交互训练 ${ }^{[19]}$ 。根据踝关节损伤的程 度, 选择不同的康复训练模式; 在被动康复训练中, 踝康复机器人根据医师的康复路径规划, 带动踝关 节进行单自由度、多自由度运动。当踝关节具备一 定的运动能力时, 采用主动模式, 踝康复机器人基 于脚掌及踝关节对动平台施加的作用力/矩, 判断患 
者的运动意图, 辅助踝关节按照意愿的路径进行运 动。随着踝关节康复效果的累积, 可选择人机交互 训练模式, 通过控制人-机之间的作用力/矩, 改变 人-机之间的运动阻抗, 训练踝关节自身运动范围和 肌肉力量。

通过建立完整的作用力/矩信息采集系统, 全面 测量人-机之间的作用力/矩, 记录踝关节不同运动 过程中的路径轨迹和产生的作用力，同时，通过机 器人路径记录功能, 复制医师康复路径, 提高了机 器人、患者及医师之间的交互能力。医师可根据采 集得到的数据, 对康复过程进行实时观测和评估, 定量分析踝关节的康复效果。

\section{2 踝康复机构的运动学分析}

运动学分析对于踝康复机器人的工作空间分 析、运动轨迹规划和性能分析具有重要意义, 因 此, 需建立踝康复机构的运动学模型, 分析其运动 学特征。

\section{1 踝康复机构的位置逆解}

建立踝康复机构运动学坐标系统, 如图 6 所示, 在转动中心处, 建立定坐标系 $\{0\}$ 和动坐标系 $\{4\}$, $x_{0} 、 y_{0} 、 z_{0}$ 分别与转动副 $\mathrm{R}_{2} 、 \mathrm{R}_{3} 、 \underline{\mathrm{R}}_{1}$ 转动轴线重合。 初始位置时坐标系 $\{0\}$ 和 $\{4\}$ 重合, 虎克副 $\mathrm{U}_{i}$ 的第 1 根轴线 $u_{1, i}$ 与 $x_{0}$ 平行, 第 2 根轴线 $u_{2, i}$ 与 $y_{0}$ 平行; 坐标系 $\{1, i\}$ 建立在 $\mathrm{U}_{i}$ 几何中心 $a_{i}$ 处, 轴 $x_{1, i}$ 与 $u_{1, i}$ 重合, 在踝康复机构初始位形下轴 $y_{1, i}$ 与 $u_{2, i}$ 重合; 坐标系 $\{2, i\}$ 与移动副 $\underline{\mathrm{P}}_{i}$ 固连, 原点 $o_{2, i}$ 与 $o_{1, i}$ 重合, 轴 $y_{2, i}$ 与 $u_{2, i}$ 重合, 轴 $z_{2, i}$ 与移动副 $\underline{\mathrm{P}_{i}}$ 的轴线重合; 坐标系 $\{3, i\}$ 建立在球副几何中心 $c_{i}$ 处, 轴线与坐标 系 $\{2, i\}$ 平行, 其中 $i=1,2$ 。 $o^{\prime}$ 为 $z_{0}$ 轴与基座的交点,

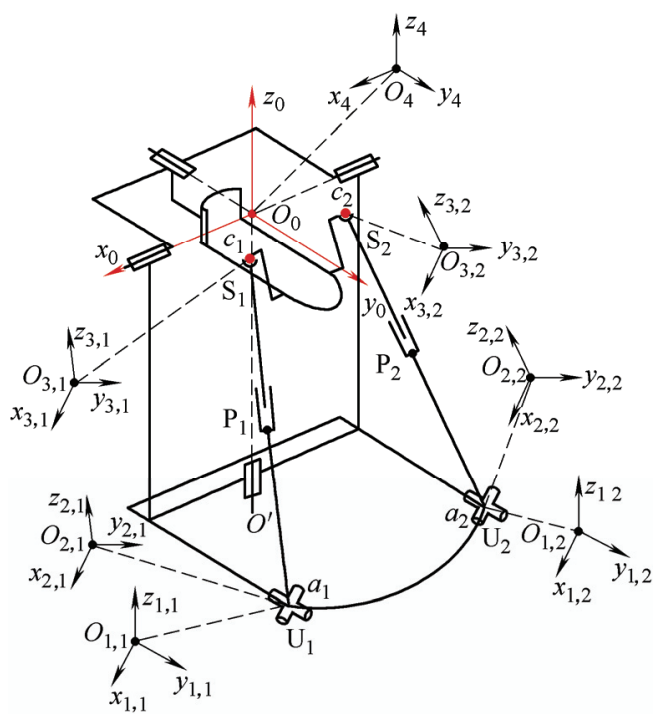

图 6 踝康复机构的坐标系统 $l_{0} 、 l_{1, i} 、 l_{2, i}$ 分别为线段 $o_{0} o^{\prime} 、 o^{\prime} a_{i} 、 a_{i} c_{i}$ 的长度, $\theta_{1, i} 、$ $\theta_{2, i}$ 分别为胡克副 $\mathrm{U}_{i}$ 绕 $u_{1, i}$ 和 $u_{2, i}$ 转过的角度, $\theta_{3}$ 为 动平台绕 $z_{0}$ 轴转过的角度。

基于踝康复机构的自由度特性, 采用 $Z-X-Y$ 欧 拉角表示动平台位姿, $\alpha 、 \beta$ 和 $\gamma$ 分别为趾屈/背伸角、 内翻/外翻角及内收/外展角, 即动平台绕 $x_{0}$ 轴(趾屈 为正/背伸为负)、 $y_{0}$ 轴(内翻为正/外翻为负)和 $z_{0}$ 轴 (内收为正/外展为负)的旋转角度。当上述旋转角度 已知时, 动坐标系 $\{4\}$ 相对于定坐标系 $\{0\}$ 的旋转变 换矩阵 ${ }_{4}^{0} \boldsymbol{R}$ 可表示为

$$
{ }_{4}^{0} \boldsymbol{R}=\boldsymbol{R}_{z}(\gamma) \cdot \boldsymbol{R}_{x}(\alpha) \cdot \boldsymbol{R}_{y}(\beta)
$$

给定踝康复机构动平台在空间的姿态角 $\alpha 、 \beta$ 、 $\gamma$, 求解电动推杆和电动机的输入量, 称之为位置逆 解问题 ${ }^{[20]}$, 位置逆解对工作空间分析和精确控制均 有重要意义。

采用封闭矢量环法求解踝康复机构的位置逆 解, 如图 7 所示, UPS 支链球副 $\mathrm{S}_{i}$ 中心点 $c_{i}$ 的位置 矢量为

$$
{ }^{0} \boldsymbol{r}_{c, i}={ }^{0} \boldsymbol{r}_{o, 4}+{ }_{4}^{0} \boldsymbol{R} \cdot{ }^{4} \boldsymbol{r}_{c, i}
$$

$$
{ }^{0} \boldsymbol{r}_{c, i}={ }^{0} \boldsymbol{r}_{a, i}+l_{2, i} \cdot \boldsymbol{z}_{2, i}={ }^{0} \boldsymbol{r}_{a, i}+l_{2, i} \cdot{ }_{2}^{0} \boldsymbol{R} \cdot\left(\begin{array}{l}
0 \\
0 \\
1
\end{array}\right)
$$

式中, ${ }_{2}^{0} \boldsymbol{R}$ 是坐标系 $\{2\}$ 相对于 $\{0\}$ 的旋转变换矩阵, ${ }^{0} \boldsymbol{r}_{a, i,} 、{ }^{0} \boldsymbol{r}_{c, i} 、{ }^{0} \boldsymbol{r}_{o, 4}$ 分别是点 $a_{i} 、 c_{i} 、 o_{4}$ 在坐标系 $\{0\}$ 中的 位置矢量, ${ }^{4} \boldsymbol{r}_{c, i}$ 是点 $c_{i}$ 在坐标系 $\{4\}$ 中的位置矢量,

$$
\begin{gathered}
{ }_{2}^{0} \boldsymbol{R}_{i}={ }_{1}^{0} \boldsymbol{R}_{i} \cdot{ }_{2}^{1} \boldsymbol{R}_{i} \\
{ }_{2}^{1} \boldsymbol{R}_{i}=\left[\begin{array}{ccc}
\mathrm{c} \theta_{2, i} & 0 & \mathrm{~s} \theta_{2, i} \\
\mathrm{~s} \theta_{1, i} \cdot \mathrm{s} \theta_{2, \mathrm{i}} & \mathrm{c} \theta_{1, i} & -\mathrm{c} \theta_{2, i} \cdot \mathrm{s} \theta_{1, i} \\
-\mathrm{c} \theta_{1, i} \cdot \mathrm{s} \theta_{2, i} & \mathrm{~s} \theta_{1, i} & \mathrm{c} \theta_{1, i} \cdot \mathrm{c} \theta_{2, i}
\end{array}\right]
\end{gathered}
$$

式中， $\mathrm{c} 、 \mathrm{~s}$ 分别为 $\cos 、 \sin$ 的简称。

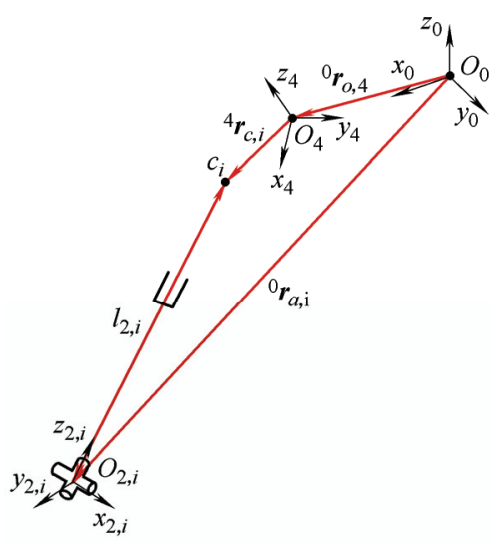

图 7 位置逆解矢量环

由式(3)、(4)得 


$$
l_{2, i}=\left|{ }^{0} \boldsymbol{r}_{o, 4}+{ }_{4}^{0} \boldsymbol{R} \cdot{ }^{4} \boldsymbol{r}_{c, i}-{ }^{0} \boldsymbol{r}_{a, i}\right|
$$

由踝康复机构的运动性质得

$$
\theta_{3}=\gamma
$$

通过求解式(5)、(6), 可得到电动推杆行程 $l_{2,1}$ 、 $l_{2,2}$ 和步进电动机转角 $\theta_{3}$ 。

\section{2 踝康复机构的雅可比矩阵}

球副 $\mathrm{S}_{i}$ 中心点 $c_{i}$ 的速度 $v_{c, i}$ 可表示为

$$
\boldsymbol{v}_{c, i}=\boldsymbol{v}_{o, 4}+\boldsymbol{\omega} \times\left({ }_{4}^{0} \boldsymbol{R} \cdot{ }^{4} \boldsymbol{r}_{c, i}\right)
$$

将 $v_{c, i}$ 分别沿坐标系 $\{3, i\}$ 的各坐标轴分解可得

$$
\begin{aligned}
& {\left[\begin{array}{l}
\boldsymbol{v}_{x c, i} \\
\boldsymbol{v}_{y c, i} \\
\boldsymbol{v}_{z c, i}
\end{array}\right]=\left[\begin{array}{l}
\boldsymbol{x}_{3, i} \\
\boldsymbol{y}_{3, i} \\
\boldsymbol{z}_{3, i}
\end{array}\right] \boldsymbol{v}_{c, i}} \\
& \boldsymbol{x}_{3, i}=\boldsymbol{x}_{2, i}={ }_{2}^{0} \boldsymbol{R}_{i} \cdot\left(\begin{array}{lll}
1 & 0 & 0
\end{array}\right)^{\mathrm{T}} \\
& \boldsymbol{y}_{3, i}=\boldsymbol{y}_{2, i}={ }_{2}^{0} \boldsymbol{R}_{i} \cdot\left(\begin{array}{lll}
0 & 1 & 0
\end{array}\right)^{\mathrm{T}} \\
& \boldsymbol{z}_{3, i}=\boldsymbol{z}_{2, i}={ }_{2}^{0} \boldsymbol{R}_{i} \cdot\left(\begin{array}{lll}
0 & 0 & 1
\end{array}\right)^{\mathrm{T}}
\end{aligned}
$$

由驱动支链的结构特点及式(8)可得约束支链 $u_{1, i}, u_{2, i}, \mathrm{P}_{i}$ 的速度为

$$
\left[\begin{array}{c}
\boldsymbol{v}_{u 1, i} \\
\boldsymbol{v}_{u 2, i} \\
\boldsymbol{v}_{\mathrm{P}, i}
\end{array}\right]=\left[\begin{array}{lll}
\left(l_{2, i} \boldsymbol{x}_{1, i} \cdot \boldsymbol{x}_{2, i}\right)^{-1} & & \\
& l_{2, i}{ }^{-1} & \\
& & 1
\end{array}\right] \cdot\left[\begin{array}{l}
\boldsymbol{v}_{y c, i} \\
\boldsymbol{v}_{x c, i} \\
\boldsymbol{v}_{z c, i}
\end{array}\right]
$$

将式(7)代入式(8)、(9)化简得

$$
\begin{aligned}
& {\left[\begin{array}{c}
\boldsymbol{v}_{u 1, i} \\
\boldsymbol{v}_{u 2, i} \\
\boldsymbol{v}_{\mathrm{P}, i}
\end{array}\right]=\left[\begin{array}{c}
\left(l_{2, i} \boldsymbol{x}_{1, i} \cdot \boldsymbol{x}_{2, i}\right)^{-1} \cdot \boldsymbol{y}_{3, i}{ }^{\mathrm{T}} \cdot \boldsymbol{Q}_{i} \\
l_{2, i}{ }^{-1} \cdot \boldsymbol{x}_{3, i}{ }^{\mathrm{T}} \cdot \boldsymbol{Q}_{i} \\
\boldsymbol{z}_{3, i}{ }^{\mathrm{T}} \cdot \boldsymbol{Q}_{i}
\end{array}\right] \cdot \boldsymbol{\omega}} \\
& \boldsymbol{Q}_{i}=\left[\begin{array}{ccc}
0 & \boldsymbol{E}_{z}^{\mathrm{T}}\left({ }_{4}^{0} \boldsymbol{R} \cdot{ }^{4} \boldsymbol{r}_{c, i}\right) & -\boldsymbol{E}_{z}^{\mathrm{T}}\left({ }_{4}^{0} \boldsymbol{R} \cdot{ }^{4} \boldsymbol{r}_{c, i}\right) \\
-\boldsymbol{E}_{z}^{\mathrm{T}}\left({ }_{4}^{0} \boldsymbol{R} \cdot{ }^{4} \boldsymbol{r}_{c, i}\right) & 0 & \boldsymbol{E}_{z}^{\mathrm{T}}\left({ }_{4}^{0} \boldsymbol{R} \cdot{ }^{4} \boldsymbol{r}_{c, i}\right) \\
\boldsymbol{E}_{z}^{\mathrm{T}}\left({ }_{4}^{0} \boldsymbol{R} \cdot{ }^{4} \boldsymbol{r}_{c, i}\right) & -\boldsymbol{E}_{z}^{\mathrm{T}}\left({ }_{4}^{0} \boldsymbol{R} \cdot{ }^{4} \boldsymbol{r}_{c, i}\right) & 0
\end{array}\right] \\
& \boldsymbol{E}_{x}=\left(\begin{array}{l}
1 \\
0 \\
0
\end{array}\right) \quad \boldsymbol{E}_{y}=\left(\begin{array}{l}
0 \\
1 \\
0
\end{array}\right) \quad \boldsymbol{E}_{z}=\left(\begin{array}{l}
0 \\
0 \\
1
\end{array}\right) \\
& {\left[\begin{array}{l}
\boldsymbol{v}_{\mathrm{P}, 1} \\
\boldsymbol{v}_{\mathrm{P}, 2} \\
\boldsymbol{v}_{\mathrm{R}, 1}
\end{array}\right]=\left[\begin{array}{ccc} 
& \boldsymbol{z}_{3,1}{ }^{\mathrm{T}} \cdot \boldsymbol{Q}_{1} & \\
\boldsymbol{z}_{3,2}{ }^{\mathrm{T}} \cdot \boldsymbol{Q}_{2} & \\
0 & 0 & 1
\end{array}\right] \cdot \boldsymbol{\omega}=\boldsymbol{J}_{3}{ }^{-1} \cdot \boldsymbol{\omega}}
\end{aligned}
$$

雅可比矩阵描述了动平台 3 个轴向转动角速度 $\omega$ 与电动推杆速度 $\boldsymbol{v}_{\mathrm{P}, 1}, \boldsymbol{v}_{\mathrm{P}, 2}$ 、电动机速度 $\boldsymbol{v}_{\mathrm{R}, 1}$ 之间的 映射关系。由式(11)可知, 输入端含 2 个线性运动, 而输出端为 3 个旋转运动。在评价踝康复机器人运 动性能时, 映射的放大倍数会随着量纲的改变而改
变。为了正确合理地评价其运动学性能指标, 需要 引入量纲一雅可比矩阵

$$
\begin{aligned}
& \boldsymbol{J}=\left[\begin{array}{ccc} 
& \boldsymbol{z}_{3,1}{ }^{\mathrm{T}} \cdot \boldsymbol{Q}_{1}{ }^{*} & \\
& \boldsymbol{z}_{3,2}{ }^{\mathrm{T}} \cdot \boldsymbol{Q}_{2}{ }^{*} & \\
0 & 0 & 1
\end{array}\right]^{-1} \\
& \boldsymbol{Q}_{i}^{*}=\left[\begin{array}{ccc}
0 & \boldsymbol{E}_{z}^{\mathrm{T}}\left({ }_{4}^{0} \boldsymbol{R} \cdot{ }^{4} \boldsymbol{r}_{c, i}^{0}\right) & -\boldsymbol{E}_{z}^{\mathrm{T}}\left({ }_{4}^{0} \boldsymbol{R} \cdot{ }^{4} \boldsymbol{r}_{c, i}^{0}\right) \\
-\boldsymbol{E}_{z}^{\mathrm{T}}\left({ }_{4}^{0} \boldsymbol{R} \cdot{ }^{4} \boldsymbol{r}_{c, i}^{0}\right) & 0 & \boldsymbol{E}_{z}^{\mathrm{T}}\left({ }_{4}^{0} \boldsymbol{R} \cdot{ }^{4} \boldsymbol{r}_{c, i}^{0}\right) \\
\boldsymbol{E}_{z}^{\mathrm{T}}\left({ }_{4}^{0} \boldsymbol{R} \cdot{ }^{4} \boldsymbol{r}_{c, i}^{0}\right) & -\boldsymbol{E}_{z}^{\mathrm{T}}\left({ }_{4}^{0} \boldsymbol{R} \cdot{ }^{4} \boldsymbol{r}_{c, i}^{0}\right) & 0
\end{array}\right] \\
& { }^{4} \boldsymbol{r}_{c, i}^{0}=k \frac{{ }^{4} \boldsymbol{r}_{c, i}}{\left|{ }^{4} \boldsymbol{r}_{c, i}\right|}
\end{aligned}
$$

${ }^{4} \boldsymbol{r}^{0}$ 为球副 $\mathrm{S}_{i}$ 相对于动平台的量纲一矢径; $k$ 为比例 因子, $k$ 越大则旋转量所占比重越大, 一般取 $k=1$ 。

\section{3 踝康复机器人工作空间分析}

踝康复机器人的有效工作空间是指满足装置整 体尺寸和结构干涉, 动平台可达位置和姿态的集合, 其大小是衡量机器人性能的重要指标, 直接影响其 实际应用价值。

\section{1 踝关节生理运动空间的测量}

由于性别、年龄和身体状况的不同，踝关节的 生理运动空间不尽相同，文献[21]测量了踝关节做 单轴运动时的运动范围, 然而现有文献均未对踝关 节的全域运动空间进行完整描述。为了更好地评价 人-机运动空间的吻合度，设计检测试验平台，测量 踝关节在全范围自主运动时的生理运动空间。

将踝康复机器人的 UPS 支链拆开, 得到由 3 个 转动副 $\left(R_{1} 、 R_{2} 、 R_{3}\right)$ 串联而成的踝关节运动空间的 测量装置, 如图 8a 所示, 编码器实时检测踝关节 3 个轴向的运动角度。利用弹性绑带将被测者的脚掌 绑缚于上平台，保证人-机之间具有较好的连接强度 和作用力传递性能, 调节脚掌位置, 确保人-机转动 中心近似重合, 如图 $8 \mathrm{~b}$ 所示。为了降低大腿和小腿 对踝关节运动的影响，使用木质夹具和弹性绑带对 大腿和小腿进行绑缚固定, 初始位置时大腿平面脚平面-动平台三者保持平行且水平。踝关节分别完 成全范围自主运动和环转运动, 自主运动是在不同 内收/外展面内完成趾屈/背伸和内翻/外翻运动, 环 转运动为踝关节沿生理运动空间边界依次做背伸内翻-趾屈-外翻的复合运动。参与测量的人员包含 男性 9 名(平均年龄 25.89 岁, 平均身高 $175.33 \mathrm{~mm}$, 平均体重 $66.11 \mathrm{~kg}$ ) 和女性 3 名(平均年龄 26 岁, 平 均身高 $162 \mathrm{~mm}$, 平均体重 $55 \mathrm{~kg}$ ), 测量的结果取各 
组数据的平均值。

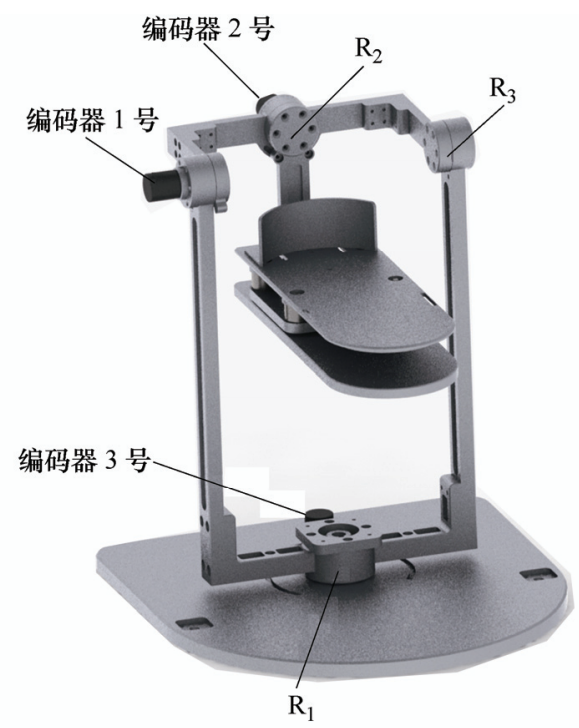

(a) 测量装置

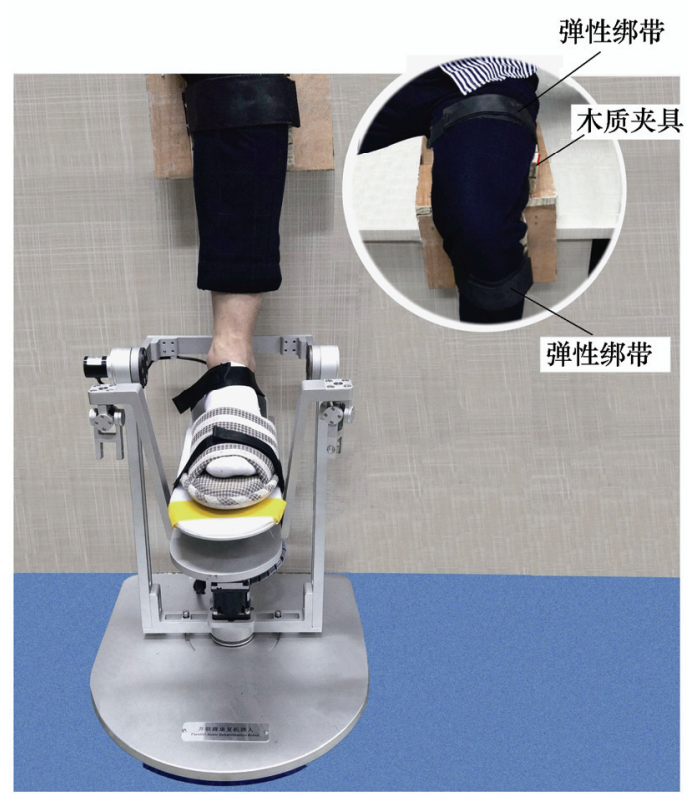

(b) 测量试验

图 8 踝关节生理运动空间测量

踝关节完成全范围自主运动时的生理运动空间 (PMS)如图 9 所示, 当脚踝做复合运动时其单轴的 运动范围要小于初始位置时单轴运动的范围, 例如, 当内收/外展角 $\gamma_{\mathrm{an}}=0^{\circ}$ 、趾屈/背伸角 $\alpha_{\mathrm{an}}=0^{\circ}$ 时, $-14.9^{\circ}<\beta_{\mathrm{an}}<17.8^{\circ}$, 当 $\gamma_{\mathrm{an}}=20^{\circ} 、 \alpha_{\mathrm{an}}=0^{\circ}$ 时, $-14.29^{\circ}<\beta_{\mathrm{an}}<9.8^{\circ}$, 而当 $\gamma_{\mathrm{an}}=20^{\circ} 、 \alpha_{\mathrm{an}}=20^{\circ}$ 时, $-3.4^{\circ}<\beta_{\mathrm{an}}<9.5^{\circ}$, 随着 $\alpha_{\mathrm{an}}$ 和 $\gamma_{\mathrm{an}}$ 的增加, 内翻/外翻角 $\beta_{\mathrm{an}}$ 呈非对称减小趋势。踝关节全范围自主运动空间 的测量对踝康复机器人的运动边界设定、主被动康 复训练和运动性能评价提供参数支撑。

踝关节做环转运动时各运动角度如图 10 所示, 在环转运动中, 内收/外展角 $\gamma_{\mathrm{an}}$ 与内翻/外翻角 $\beta_{\mathrm{an}}$

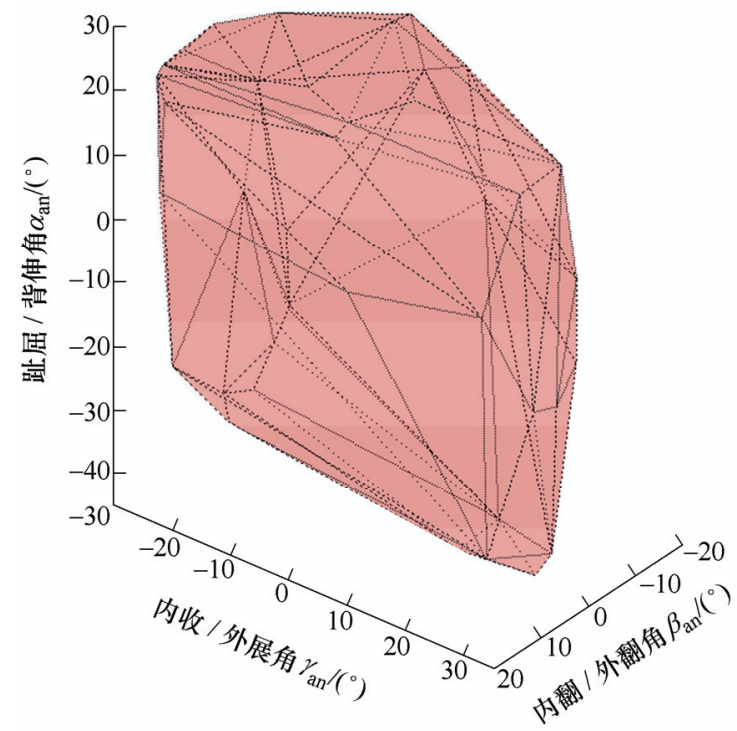

图 9 踝关节的生理运动空间 PMS

呈正弦曲线变化, 当 $\left|\alpha_{\mathrm{an}}\right|$ 达到最大值时, $\beta_{\mathrm{an}}=\gamma_{\mathrm{an}}=0^{\circ}$, 当 $\alpha_{\mathrm{an}}=0^{\circ}$ 时, $\left|\beta_{\mathrm{an}}\right|$ 与 $\left|\gamma_{\mathrm{an}}\right|$ 达到最大值, 且环转运动 与全范围自主运动的单轴运动范围保持一致, 验证 了生理运动空间测量的准确性。此外, 发现踝关节 运动的极限姿态是三种典型运动的叠加, 因此有必 要进行踝关节复合运动的康复训练。

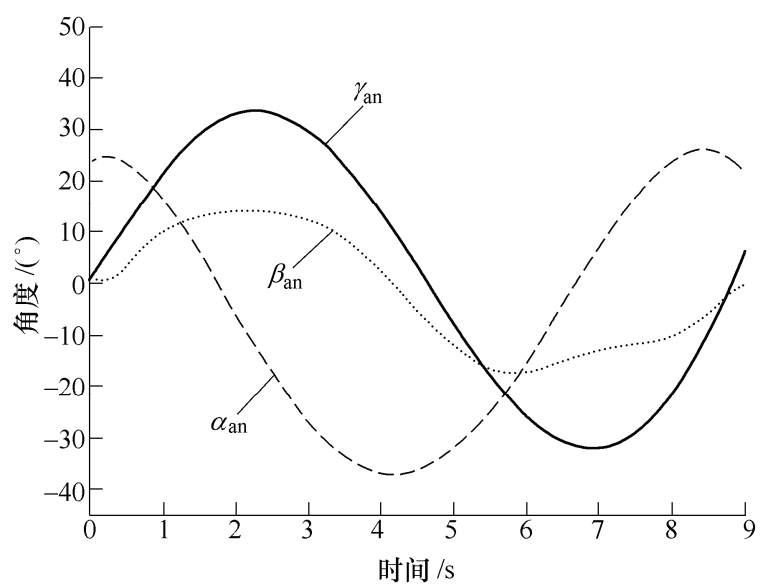

图 10 环转运动中各踝关节角度曲线

\section{2 踝康复机器人的工作空间}

踝康复机器人仅具有 3 个转动自由度, 将其工 作空间定义为：满足驱动与结构参数的条件下，动 平台所有姿态的集合。踝康复机器人的结构参数如 表 2 所示。

表 2 踝康复机器人的结构参数

\begin{tabular}{cc}
\hline 参数 & 数值 $/ \mathrm{mm}$ \\
\hline 线段 $o^{\prime} a_{i}$ 长度 $l_{1, i}$ & 192 \\
线段 $o_{0} o^{\prime}$ 长度 $l_{0}$ & 421 \\
中心点 $c_{1}$ 坐标值 & $(130,129.5,-12.5)$ \\
中心点 $c_{2}$ 坐标值 & $(-130,129.5,-12.5)$ \\
几何中心 $a_{1}$ 坐标值 & $(175,80,-421)$ \\
几何中心 $a_{2}$ 坐标值 & $(-175,80,-421)$ \\
\hline
\end{tabular}


限定移动副 $\underline{\mathrm{P}}_{i}$ 的杆长 $l_{2, i}$ 变化范围为 255 $\mathrm{mm} \leqslant l_{2, i} \leqslant 405 \mathrm{~mm}$, 虎克副 $\mathrm{U}_{i}$ 的转角范围为 $-35^{\circ} \leqslant \theta_{1, i} \leqslant 35^{\circ}$, 借助 Matlab 软件利用边界搜索法 对踝康复机构的工作空间进行仿真分析, 得到其理 论工作空间(TWS), 如图 11 所示。背伸角 $\alpha_{\mathrm{th}}<20^{\circ}$ 时, 理论工作空间近似为矩形, $\alpha_{\mathrm{th}}>20^{\circ}$ 时, 随着 $\alpha_{\mathrm{th}}$ 的增加, 内翻/外翻角 $\beta_{\mathrm{th}}$ 和内收/外展角 $\gamma_{\mathrm{th}}$ 呈非对称 减小趋势, 理论工作空间 TWS 能够完全包裹踝关 节生理运动空间 PMS。TWS 的单轴运动范围为 $-46.2^{\circ}<\alpha_{\mathrm{th}}<30^{\circ},-23.5^{\circ}<\beta_{\mathrm{th}}<23.5^{\circ},-36^{\circ}<\gamma_{\mathrm{th}}<36^{\circ}$, 均 大于踝关节单轴运动范围。

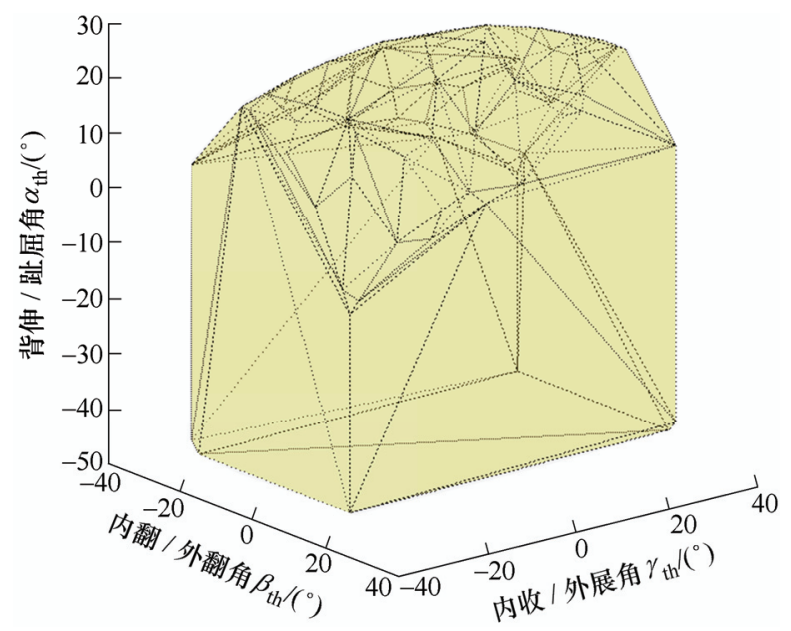

图 11 踝康复机构的理论工作空间 TWS

踝康复机器人具有 3 条驱动支链, 通过控制系 统驱动关节 $\underline{P}_{1} 、 \underline{P}_{2} 、 \underline{R}_{1}$, 使其在工作空间内完成主 动运动, 借助编码器测量关节 $\underline{R}_{1} 、 R_{2}$ 及 $R_{3}$ 的运动 范围, 可得到其有效工作空间(RWS), 如图 12 所示。 当 $-35^{\circ}<\alpha_{\mathrm{ro}}<10^{\circ}$ 时, RWS 近似为矩形, $\alpha_{\mathrm{ro}}>10^{\circ}$ 和 $\alpha_{\mathrm{ro}}<-35^{\circ}$ 时, 随着 $\left|\alpha_{\mathrm{ro}}\right|$ 的增加, 内翻/外翻角 $\beta_{\mathrm{th}}$ 和内 收/外展角 $\gamma_{\mathrm{th}}$ 呈减小趋势, 由于机器人杆长参数、 零部件的体积及干涉, 踝康复机器人的有效工作空

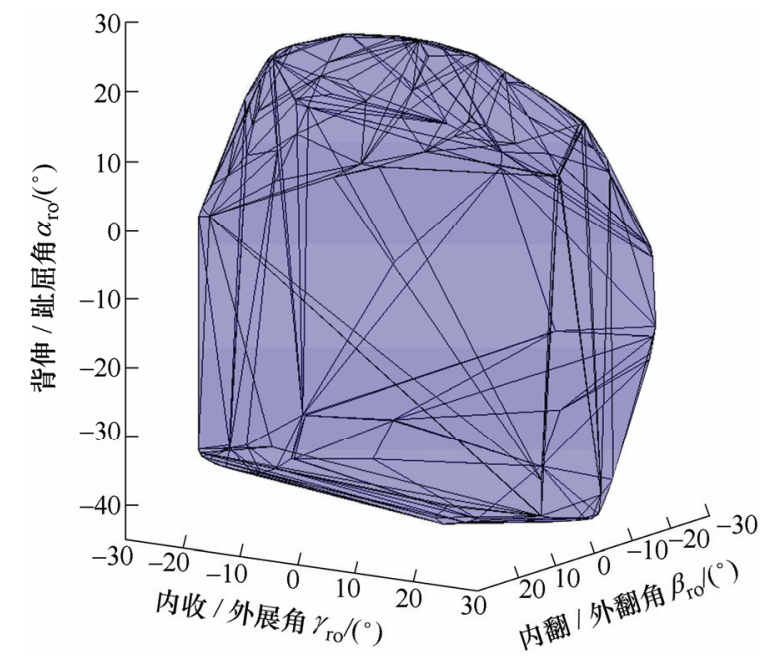

图 12 踝康复机器人的有效工作空间 RWS
间 RWS 小于其理论工作空间 TWS。基于踝关节的 生理运动空间 PMS, 将踝康复机器人的有效工作空 间 RWS 与之对比分析, 两者进行重合分布, 如图 13 所示, RWS 在内收/外展和趾屈/背伸方向能够完 全满足 PMS, RWS 单轴运动范围大于 PMS 单轴运 动范围; 为了更加清晰地观察两者的差异, 将工作 空间投影至三坐标平面, 如图 14 所示。

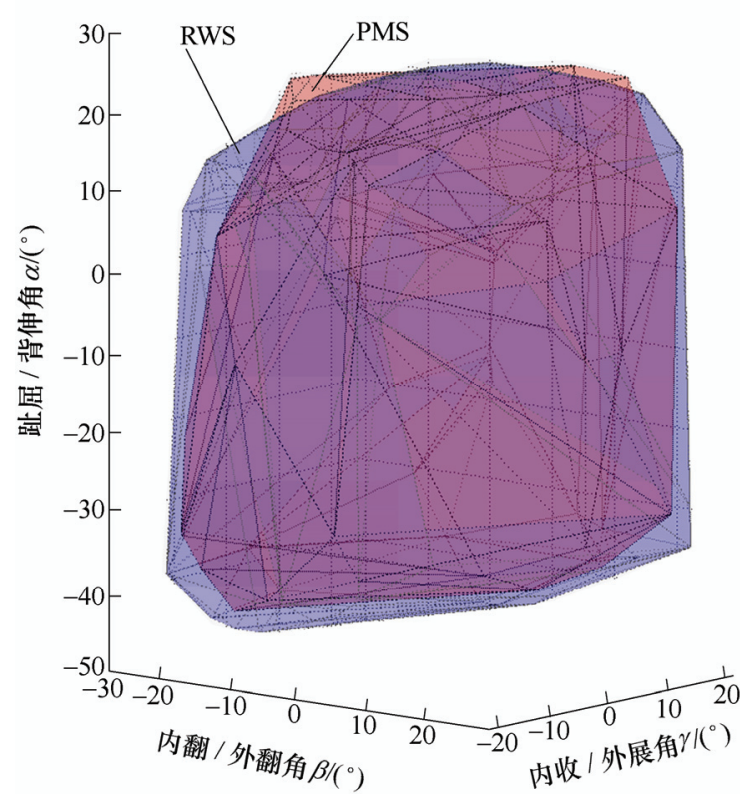

图 13 人-机运动空间对比

经对比分析可知, 人-机运动空间高度吻合, 踝 康复机器人能够全域范围内满足踝关节运动。定义 人-机运动空间的吻合度为人-机运动空间重叠区域 与踝关节生理运动范围之比, 经分析和计算可得, 人-机运动空间吻合度可达 $95 \%$ 。图 $14 \mathrm{~b}$ 的人-机运 动边界处出现较小的差异, 即在 $\alpha<-10^{\circ}$ 且 $|\beta|>5^{\circ}$ 时, 装置的内翻/外翻范围小于踝关节的相应运动范围, 其原因为电动推杆的行程不足所导致, 而其行程受 限于踝康复机器人的结构干涉和整体尺寸。进一

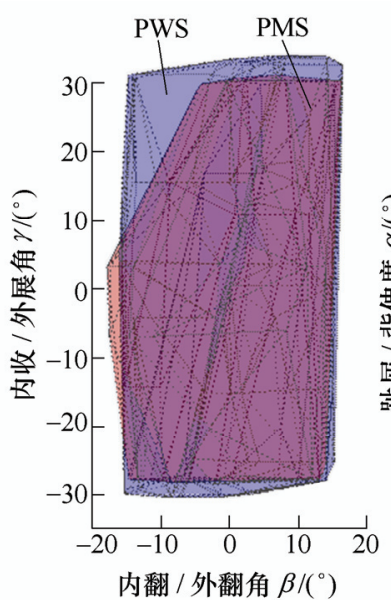

(a) $y_{0}-z_{0}$ 面投影

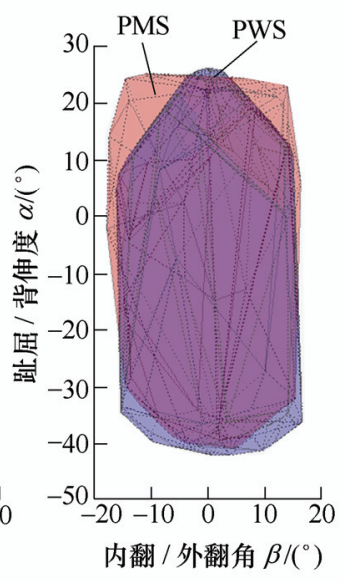

(b) $y_{0}-x_{0}$ 面投影 


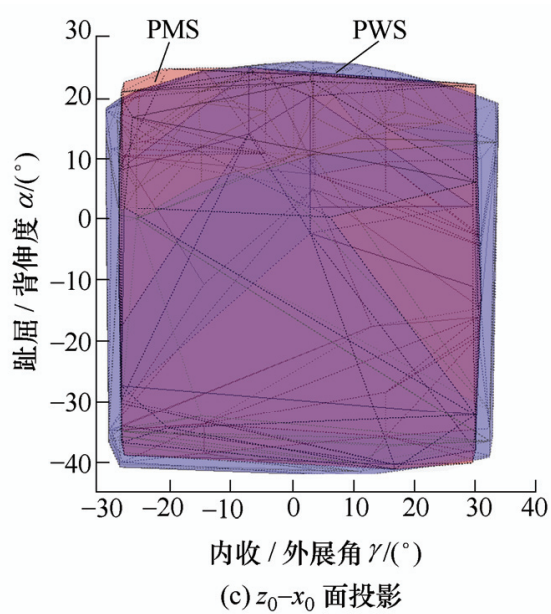

图 14 人-机运动空间在坐标系 $\{0\}$ 内的投影

步, 比较人-机单轴运动范围, 如表 3 所示, 通过 分析可知, 踝康复机器人能够完全满足踝关节单轴 运动需求。

表 3 踝关节与踝康复机器人的运动范围

\begin{tabular}{ccc}
\hline 运动类型 & 踝关节范围 $\left./{ }^{\circ}\right)$ & 踝康复机器人范围 $/\left({ }^{\circ}\right)$ \\
\hline 背伸 & 24.89 & 25.92 \\
趾屈 & 41.11 & 42.24 \\
内翻 & 16.28 & 16.46 \\
外翻 & 15.80 & 16.11 \\
内收 & 30.96 & 33.71 \\
外展 & 28.40 & 30.49 \\
\hline
\end{tabular}

\section{4 踝康复机器人的运动性能评价}

雅可比矩阵用于表征力和速度在关节空间与操 作空间的映射关系, 其代数特征值(条件数、奇异值、 行列式等)可作为踝康复机器人运动学性能的重要 评价指标 ${ }^{[22]}$ 。

\section{1 可操作度分析}

可操作度是反映机器人动平台在位移和姿态方 面可操作能力的度量, 亦是评价其在某位姿下灵活 性的指标 ${ }^{[23]}$ 。当机器人处于奇异位形时, 可操作度 $W=0$, 将失去沿某一方向的活动能力, 灵活性也最 差, 因此, 应当尽量避免接近奇异位形 ${ }^{[24]}$ 。当机器 人处于非奇异位形时, 可操作度为

$$
W=\sqrt{\operatorname{det}\left(\boldsymbol{J} \cdot \boldsymbol{J}^{\mathrm{T}}\right)}
$$

在踝关节生理运动空间内, 以 $\alpha 、 \beta$ 为连续变量, 根据踝关节运动频率的分布, 内收/外展角 $\gamma$ 分别取 $-10^{\circ} 、 0^{\circ}$ 及 $10^{\circ}$, 可得到在不同的姿态下踝康复机器 人的可操作度, 如图 15 所示。

由图 15 可知, 踝康复机器人的可操作度不存在 零值和突变, 且变化平稳连续, 表明在工作空间内

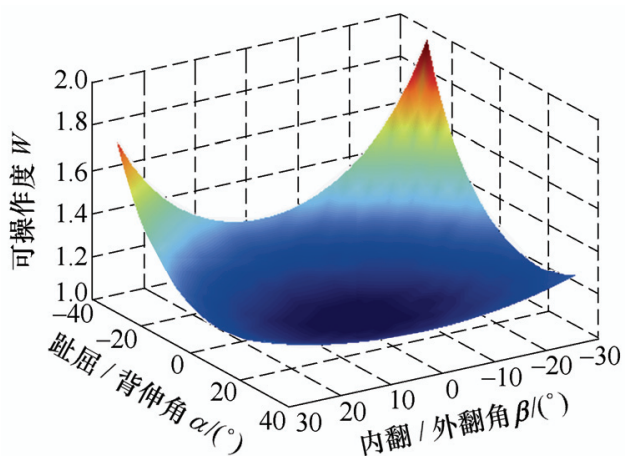

(a) 内收 / 外展角 $\gamma=10^{\circ}$

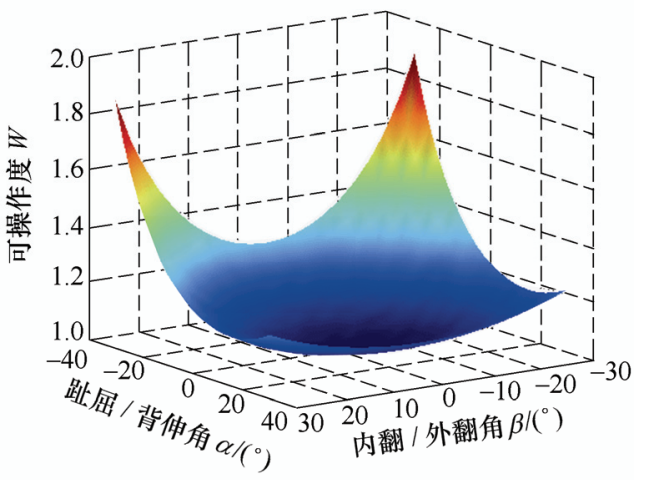

(b) 内收 / 外展角 $\gamma=0^{\circ}$

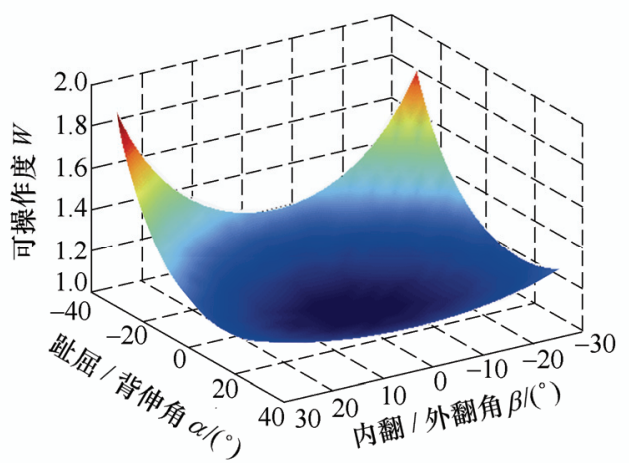

(c) 内收 / 外展角 $\gamma=-10^{\circ}$

图 15 踝康复机器人的可操作度 $W$

无奇异位形且可操作性良好。在实际应用中，由于 可操作度难以描述输入偏差相对于输出偏差的影 响, 需要用雅可比矩阵的条件数对踝康复机器人的 灵巧性进一步分析与评价。

\section{2 灵巧性分析}

灵巧性是反映机器人综合运动性能优劣的重要 指标, SALISBURY 等提出采用雅可比矩阵的条件 数作为灵巧性指标 ${ }^{[25]}$ 。雅可比矩阵的条件数 $K$ 的取 值范围为 $1 \leqslant K \leqslant+\infty, K$ 值越大, 机器人的输入关节 速度变化对输出速度的影响越大, 因此, 条件数 $K$ 应尽可能地小, 当 $K=1$ 时, 机器人运动具有各向同 性, 此时灵活度最好, 当 $K=+\infty$ 时, 机器人处于奇 异位形 ${ }^{[26]}$ 。设定踝康复机器人动平台的输出角速度 为 $\omega$, 输入关节速度为 $v_{\text {in }}$, 则输入关节速度与输出 速度之间的映射关系为 


$$
\boldsymbol{\omega}=\boldsymbol{J} \cdot \boldsymbol{v}_{\text {in }}
$$

式中, $J$ 为踝康复机器人的无量纲速度雅可比矩阵。

当输入关节速度的偏差为 $\Delta v_{\text {in }}$ 时, 输出角速度 偏差为 $\Delta \omega$, 速度映射关系可表示为

$$
\omega+\Delta \omega=J \cdot\left(v_{\text {in }}+\Delta v_{\text {in }}\right)
$$

联立式(14)、(15)可得

$$
\Delta \omega=J \cdot \Delta v_{\text {in }}
$$

由式(16)得主动关节输入速度 $v_{\mathrm{in}}$ 与动平台输出 速度 $\omega$ 的相对偏差为

$$
\frac{\|\Delta \omega\|}{\|\omega\|} \leqslant\|\boldsymbol{J}\| \cdot\left\|J^{-1}\right\| \cdot \frac{\left\|\Delta v_{\text {in }}\right\|}{\left\|v_{\text {in }}\right\|}
$$

令 $K=\|\boldsymbol{J}\| \cdot\left\|J^{-1}\right\|, K$ 为雅可比矩阵 $J$ 的条件数。 图 16 绘制了内收/外展角 $\gamma$ 取 $-10^{\circ} 、 0^{\circ}$ 和 $10^{\circ}$ 时, 踝 康复机器人条件数的分布。

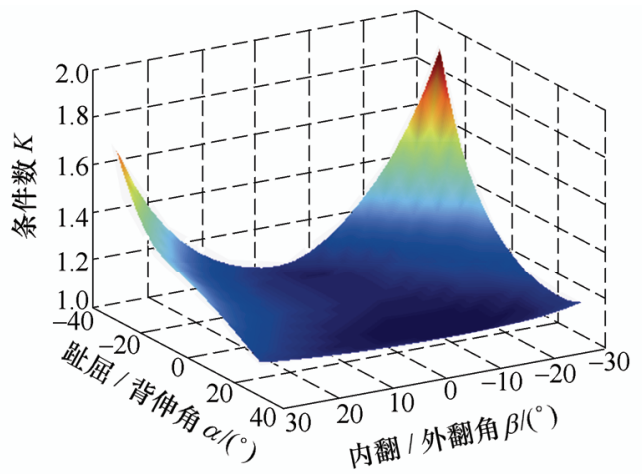

(a) 内收 / 外展角 $\gamma=10^{\circ}$

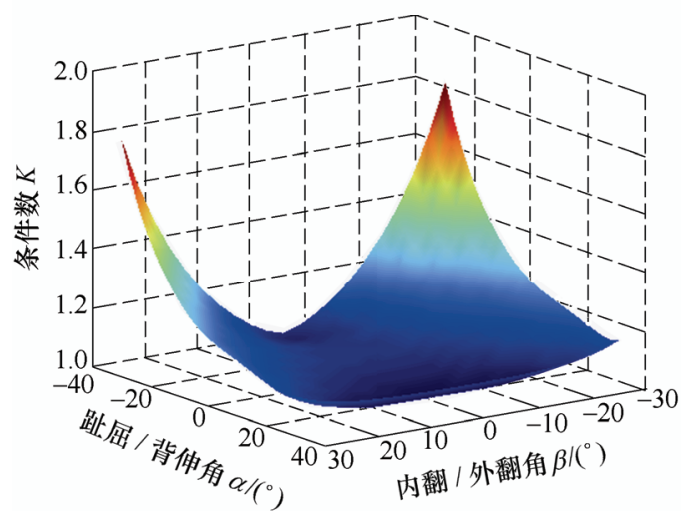

(b) 内收 / 外展角 $\gamma=0^{\circ}$

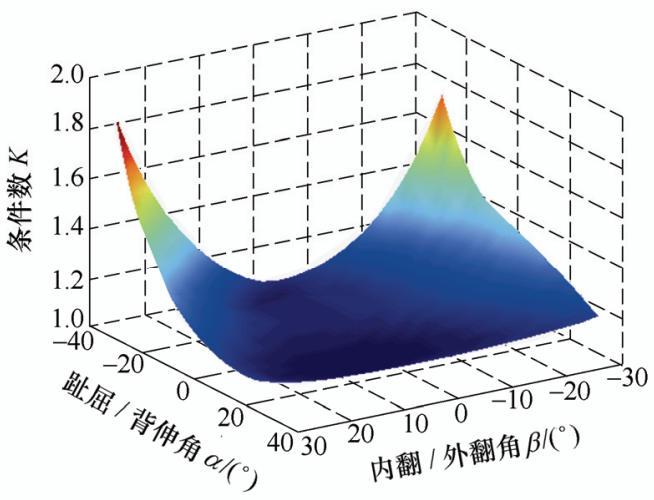

(c) 内收 / 外展角 $\gamma=-10^{\circ}$

图 16 踝康复机器人的条件数 $K$
由图 16 知, 踝康复机器人在整个工作空间内, $1.2 \leqslant K \leqslant 2.1$, 条件数 $K$ 随着各转角的不断增大而增 大，趾屈运动的条件数随角度变化幅度较大，被神 运动随角度变化较小, 当踝关节位于极限运动姿态 时, $K$ 达到极大值。条件数变化趋势平缓连续, 不 存在突变情况, 表明其在工作空间内无奇异位形且 具有较好的运动灵活性。

\section{5 结论}

（1）将踝关节的解剖结构及运动特性考虑在 内, 提出并研制 2-UPS/ $\underline{R R R}$ 型并联踝康复机器人样 机, 结构紧凑, 可保证人-机转动中心近似重合。同 时, 搭建了作用力/矩信息采集平台, 实现踝关节背 伸/跖屈、内翻/外翻和内收/外展运动的被动、主动 及人机交互训练, 提高了机器人、患者及医师之间 的交互能力。

（2）建立了踝康复机构的运动学模型, 通过求 解其位置逆解, 得到了其理论工作空间。借助机器 人样机的 3 转动自由度, 精确地测量得到踝关节全 域范围内的生理运动范围, 在踝关节环转运动时, 各踝运动角呈正/余弦曲线分布。通过机器人样机的 全范围自主运动, 记录并获得装置的有效工作空间。 通过对比分析该样机的理论和有效工作空间可知, 在关节杆长、结构干涉等尺寸约束下, 机器人有效 工作空间小于理论工作空间, 有效工作空间的测量 具有较大的实际意义。进一步, 对比分析机器人样 机的有效工作空间和踝关节生理运动范围可知, 两 者在踝全域运动范围内的吻合度可达 $95 \%$, 较好地 保障了踝关节康复所需要的运动空间。

（3）基于并联踝康复机器人的无量纲速度雅可 比矩阵, 对机器人的可操作度、灵巧性等运动学性 能进行了分析, 结果表明踝康复机器人在工作空间 内不存在奇异位形且具有良好的运动学性能。

\section{参 考 文 献}

[1] 焦爽, 间汝蕴. 本体感觉训练预防踝关节运动损伤研究 进展[J]. 中国运动医学杂志, 2009, 28(6): 713-716.

JIAO Shuang, YAN Ruyun. Research progress of proprioception training in preventing ankle injury[J]. Chinese Journal of Sports Medicine, 2009, 28(6): 713-716

[2] 边辉, 刘艳辉, 梁志成, 等. 并联 2-RRR/UPRR 踝关节 康复机器人机构及其运动学 $[\mathrm{J}]$. 机器人, 2010, 32(1): 6-12.

BIAN Hui, LIU Yanhui, LIANG Zhicheng, et al. A novel 2-RRR/UPRR robot mechanism for ankle rehabilitation 
and its kinematics[J]. Robot, 2010，32(1): 6-12.

[3] 何可, 艾坤, 谭洁, 等. 足下垂助行仪用于治疗脑卒中 后踝关节功能障碍的临床研究 $[\mathrm{J}]$. 中国康复医学杂志, 2015, 30(5): 472-475.

HE Ke, AI Kun, TAN Jie, et al. Clinical study of foot drop walking aid in the treatment of ankle dysfunction after stroke[J]. Chinese Journal of Rehabilitation Medicine, 2015, 30(5): 472-475.

[4] 陆宸照. 踝关节损伤的诊断和治疗 $[\mathrm{M}]$. 上海: 上海科 学技术文献出版社, 1998 .

LU Chenzhao. Diagnosis and treatment of ankle injury[M]. Shanghai: Shanghai Scientific and Technical Literature Publishing House, 1998.

[5] BLAYA J A , HERR H. Adaptive control of a variable-impedance ankle-foot orthosis to assist drop-foot gait[J]. IEEE Transactions on Neural Systems and Rehabilitation Engineering, 2004, 12(1): 24-31.

[6] AGRAWAL A, BANALA S K, AGRAWAL S K, et al. Design of a two degree-of-freedom ankle-foot orthosis for robotic rehabilitation[C]// International Conference on Rehabilitation Robotics, 28 June-1 July, 2005, Chicago, IL, USA. New York: IEEE, 2005: 41-44.

[7] GIRONE M J, BURDEA G C, BOUZIT M. The "Rutgers Ankle" orthopedic rehabilitation interface[J]. Proceedings of the ASME Haptics Symposium, 1999，67: 305-312.

[8] GIRONE M J, BURDEA G C, BOUZIT M, et al. A stewart platform-based system for ankle telerehabilitation[J]. Autonomous Robots，2001， 10(2): 203-212.

[9] SAGLIA J A, TSAGARAKIS N G, DAI J S, et al. A high performance 2-dof over-actuated parallel mechanism for ankle rehabilitation[C]// IEEE International Conference on Robotics and Automation, 12-17 May, 2009, Kobe, Japan. New York, IEEE, 2009: 2180-2186.

[10] DAI J S, ZHAO T, NESTER C. Sprained ankle physiotherapy based mechanism synthesis and stiffness analysis of a robotic rehabilitation device[J]. Autonomous Robots, 2004, 16(2): 207-218.

[11] 刘更谦, 高金莲, 杨四新, 等. 踝关节康复训练并联机 构构型及其运动学分析 $[\mathrm{J}]$. 机电产品开发与创新, 2005, 18(5): 13-15.

LIU Gengqian, GAO Jinlian, YANG Sixin, et al. The configuration of the ankle rehabilitation exercises parallel mechanism and its kinematics analysis[J]. Development \& Innovation of Machinery \& Electrical Products, 2005, 18(5): $13-15$.

[12] 赵铁石, 于海波, 戴建生. 一种基于 3-RSS/S 并联机构 的踝关节康复机器人 [J]. 燕山大学学报, 2005, 29(6): 471-475.
ZHAO Tieshi, YU Haibo, DAI Jiansheng. An ankle rehabilitation device based on 3-RSS/S parallel mechanism[J]. Journal of Yanshan University, 2005, 29(6): $471-475$.

[13] 边辉, 赵铁石, 田行斌, 等. 生物融合式康复机构及其 应用 $[\mathrm{J}]$. 机器人, 2010, 32(4): 470-477.

BIAN Hui, ZHAO Tieshi, TIAN Xingbin, et al. Bio-syncretic rehabilitation mechanism and its application[J]. Robot, 2010, 32(4): 470-477.

[14] WANG C, FANG Y, GUO S, et al. Design and kinematical performance analysis of a 3-RUS/RRR redundantly actuated parallel mechanism for ankle rehabilitation[J]. Journal of Mechanisms \& Robotics, 2013, 5(4): 041003.

[15] 王正义. 足踝外科学[M]. 北京: 人民卫生出版社, 2006. WANG Zhengyi. Surgery of the foot and ankle[M]. Beijing: People's Medical Publishing House, 2006.

[16] COUGHLIN M, SALTZMAN C, ANDERSON R. 曼氏 足踝外科学 $[\mathrm{M}]$. 北京: 人民卫生出版社, 2015 .

COUGHLIN M, SALTZMAN C, ANDERSON R. Mann's surgery of the foot and ankle[M]. Beijing: People's Medical Publishing House, 2015.

[17] LI Jianfeng, ZHANG Ziqiang, TAO Chunjing, et al. Structure design of lower limb exoskeletons for gait training $[\mathrm{J}]$. Chinese Journal of Mechanical Engineering. 2015, 28(5): 878-887.

[18] HOU Y L, HU X Z, ZENG D X, et al. Biomimetic shoulder complex based on 3-PSS/S spherical parallel mechanism[J]. Chinese Journal of Mechanical Engineering, 2015, 28(1): 29-37.

[19] LU Z J, LI W G, LI M J, et al. Development of a three freedoms ankle rehabilitation robot for ankle training[C]// TENCON 2015-2015 IEEE Region 10 Conference, 1-4 November, MaCao, China. New York: IEEE, 2016: $1-5$.

[20] 黄真, 赵永生, 赵铁石. 高等空间机构学 [M]. 北京: 高等教育出版社, 2006.

HUANG Zhen, ZHAO Yongsheng, ZHAO Tieshi. Advanced spatial mechanism[M]. Beijing : Higher Education Press, 2006.

[21] TSOI Y H, XIE S Q. Design and control of a parallel robot for ankle rehabiltation[J]. International Journal of Intelligent Systems Technologies \& Applications, 2008, 8(1-4): 100-113.

[22] 汪满新, 刘海涛, 黄田. 3-S(P)R 并联机构运动学性能 评价[J]. 机械工程学报，2017，53(5): 108-115.

WANG Manxin, LIU Haitao, HUANG Tian. Kinematics performance evaluation of a 3-SPR parallel manipulator[J]. Journal of Mechanical Engineering, 2017, 
53(5): 108-115.

[23] 饶青, 白师贤. 6-6 型 Stewart 机器人的可操作性分析及 其定义[J]. 机器人，1994(6): 345-349.

RAO Qing, BAI Shixian. Manipulability analysis and deeinition of 6-SPS stewart robot[J]. Robot, 1994(6): 345-349.

[24] YOSHIKAWA T. Manipulability of robotic mechanisms[J]. International Journal of Robotics Research, 1985, 4(2): 3-9.

[25] SALISBURY J K. Articulated hands: Force control and kinematic issues[J]. International Journal of Robotics Research, 1982, 1(1): 4-17.

[26] 李剑锋, 刘钧辉, 张雷雨, 等. 人机相容型肩关节康复 外骨骼机构的运动学与灵活性分析 [J]. 机械工程学报,
2018, 54(3): 46-54.

LI Jianfeng, LIU Junhui, ZHANG Leiyu, et al. Kinematics and dexterity analysis of the human-machine compatible exoskeleton mechanism for shoulder joint rehabilitation[J]. Journal of Mechanical Engineering, 2018, 54(3): 46-54.

作者简介: 李剑锋, 男, 1964 年出生, 教授, 博士研究生导师。主要研 究方向为机器人机构学和穿戴外骨骼技术。

E-mail: lijianfeng@bjut.edu.cn

张凯, 男, 1993 年出生, 硕士研究生。主要研究方向为康复机器人机 构学。

E-mail: 729827898@qq.com

张雷雨(通信作者), 男, 1988 年出生, 博士, 讲师。主要研究方向为上 肢康复机器人及下肢柔性助力设备。

E-mail: zhangleiyu1988@126.com 